\title{
The origin of dust in galaxies revisited: the mechanism determining dust content
}

\author{
Akio K. Inoue \\ College of General Education, Osaka Sangyo University, 3-1-1 Nakagaito, Daito, Osaka 574-8530, Japan \\ (Received November 8, 2010; Revised February 9, 2011; Accepted February 25, 2011; Online published February 2, 2012)
}

\begin{abstract}
The origin of cosmic dust is a fundamental issue in planetary science. This paper revisits the origin of dust in galaxies, in particular, in the Milky Way, by using a chemical evolution model of a galaxy composed of stars, interstellar medium, metals (elements heavier than helium), and dust. We start from a review of timeevolutionary equations of the four components, and then, we present simple recipes for the stellar remnant mass and yields of metal and dust based on models of stellar nucleosynthesis and dust formation. After calibrating some model parameters with the data from the solar neighborhood, we have confirmed a shortage of the stellardust-production rate relative to the dust-destruction rate by supernovae if the destruction efficiency suggested by theoretical works is correct. If the dust-mass growth by material accretion in molecular clouds is active, the observed dust amount in the solar neighborhood is reproduced. We present a clear analytic explanation of the mechanism for determining dust content in galaxies after the activation of accretion growth: a balance between accretion growth and supernova destruction. Thus, the dust content is independent of the uncertainty of the stellar dust yield after the growth activation. The timing of the activation is determined by a critical metal mass fraction which depends on the growth and destruction efficiencies. The solar system formation seems to have occurred well after the activation and plenty of dust would have existed in the proto-solar nebula.
\end{abstract}

Key words: Cosmic dust, physical processes of dust in the interstellar medium, galaxy evolution.

\section{Introduction}

Cosmic dust grains are negligible in mass in the Universe. Nevertheless, they play a significant role in many astronomical, astrophysical, and astrochemical aspects: extinction (absorption and scattering) matter of radiation, an emission source in infrared wavelengths, a coolant and a heat source in the interstellar medium (ISM) and intergalactic medium (IGM), and a site for the formation of molecules. Therefore, dust is one of the most important constituents of the Universe. Dust is also important for planetary science because grains are material for planets.

Dust grains are formed in rapidly-cooling gas of stellar outflows (Draine and Salpeter, 1977; Yamamoto and Hasegawa, 1977). We call such grains 'stardust'. Sources of the stardust are asymptotic giant branch (AGB) stars, supernovae ( $\mathrm{SNe}$ ), red supergiants, novae, Wolf-Rayet stars, and so on (e.g., Gehrz, 1989). The main source of the stardust in the present Milky Way and the Magellanic Clouds is thought to be AGB stars (Gehrz, 1989; Draine, 2009; Matsuura et al., 2009).

SNe may also produce a significant amount of stardust (Kozasa and Hasegawa, 1987; Todini and Ferrara, 2001; Nozawa et al., 2003, 2007; Schneider et al., 2004; see also Kozasa et al., 2009). Stardust from SNe was particularly important in the early Universe because the time for stars to

Copyright (C) The Society of Geomagnetism and Earth, Planetary and Space Sciences (SGEPSS); The Seismological Society of Japan; The Volcanological Society of Japan; The Geodetic Society of Japan; The Japanese Society for Planetary Sciences; TERRAPUB.

doi:10.5047/eps.2011.02.013 evolve to the AGB phase is typically about $1 \mathrm{Gyr}$, but the cosmic time in the early Universe is less than this (Morgan and Edmunds, 2003; Maiolino et al., 2004; Dwek et al., 2007; but see also Valiante et al., 2009). The 'first' stardust may have also played an important role in changing the mode of star formation from massive-star dominated to present-day Sun-like-star dominated (Schneider et al., 2003, 2006).

However, dust formation by $\mathrm{SNe}$ remains observationally controversial. The first detections of a few $M_{\odot}$ dust freshly formed, which is much larger than expected, in Cassiopeia A (Cas A) and Kepler SN remnants (SNRs) by submillimeter observations with $S C U B A$ (Dunne et al., 2003; Morgan et al., 2003) were almost contaminated by foreground dust in the ISM on the sight-lines (Krause et al., 2004; Gomez et al., 2009). Recent infrared observations with the Spiter Space Telescope and AKARI and submillimeter observations with Herschel and BLAST of Cas A and other SNRs are in agreement with theoretical expectations of 0.01-0.1 $M_{\odot}$ per one SN (Rho et al., 2008; Sakon et al., 2009; Nozawa et al., 2010; Barlow et al., 2010; Sibthorpe et al., 2010).

Once stardust grains are injected into the ISM, they are processed there. The grains in hot gas are bombarded by thermally-moving protons and sputtered (Onaka and Kamijo, 1978; Draine and Salpeter, 1979). SN shock waves probably destroy dust grains by grain-grain collisional shattering as well as sputtering (e.g., Dwek and Arendt, 1992; Jones et al., 1994, 1996; Nozawa et al., 2006; Silvia et al., 2010). This destruction process is widely accepted and ob- 
servational evidence of the destruction has been found in several SNRs, especially with the Spitzer Space Telescope (Arendt et al., 1991, 2010; Borkowski et al., 2006; Williams et al., 2006; Dwek et al., 2008; Sankrit et al., 2010; but see Mouri and Taniguchi, 2000).

Assuming the destruction efficiency predicted theoretically, the life-time of dust grains is found to be of the order of $100 \mathrm{Myr}$ (McKee, 1989; Draine, 1990; Jones et al., 1994, 1996). On the other hand, the injection time of stardust is of the order of 1 Gyr (e.g., Gehrz, 1989). Thus, another efficient channel of dust formation is required to maintain dust content in galaxies. The most plausible mechanism is accretion growth in the ISM (Draine, 1990, 2009); in dense molecular clouds, atoms and molecules of some refractory elements and compounds accrete onto pre-existing grains and may change from the gas phase to the solid phase. Note that, unlike the sticking growth of grains well studied in protoplanetary disks, this accretion growth causes an increase in dust mass. This type of growth is favored to explain the observed depletions of some elements in the gas phase of the ISM relative to solar abundance. The correlation between the degree of depletion and the density in the ISM particularly suggests this process (e.g., Savage and Sembach, 1996; Jenkins, 2009). It is also suggested that an efficient growth is required to explain the massive dust mass observed in the early Universe (Michałowski et al., 2010).

Since the pioneering work by Dwek and Scalo (1980), much theoretical work on dust-content evolution in galaxies has been carried out (Dwek, 1998; Edmunds and Eales, 1998; Lisenfeld and Ferrara, 1998; Hirashita, 1999a, b, c; Hirashita et al., 2002; Edmunds, 2003; Inoue, 2003; Morgan and Edmunds, 2003; Dwek et al., 2007; Calura et al., 2008; Zhukovska et al., 2008; Valiante et al., 2009; Asano et al., 2011; Dwek and Cherchneff, 2011; Gall et al., 2011a, b; Mattsson, 2011; Pipino et al., 2011). These works are based on the evolutionary model of elemental abundance in galaxies called the chemical evolution model (Tinsley, 1980 for a review) and incorporate some (or all) of the three processes of formation, destruction, and growth of dust. One of the main results from recent works is the importance of accretion growth.

This paper presents a new interpretation of the mechanism for determining dust content in galaxies. Previous works imply that the mechanism is a balance between dust destruction by SNe and accretion growth in the ISM. However, to date, this point has not been discussed clearly. This paper analytically justifies this implication. For this aim, a simple one-zone model is sufficient. In addition, we present new simple recipes describing stellar remnant mass and yields of elements and dust from state-of-the-art models of stellar nucleosynthesis and the formation of stardust.

Section 2 presents a review of the basic equations. In Section 3, we present new simple recipes of stellar remnant mass and yields. In Section 4, we calibrate some model parameters to reproduce the observed properties of the solar neighborhood. Section 5 presents our analytical interpretation of the mechanism for determining dust content in galaxies, and further discussions are presented in Section 6. Experts in this field may go straight to Section 5 which is the new result of this paper.
Throughout this paper, we call elements heavier than helium 'metal' according to the custom of astronomy. We adopt the metal mass fraction (so-called metallicity) in the Sun of $Z_{\odot}=0.02$ (Anders and Grevesse, 1989) conventionally, although recent measurements suggest a smaller value of 0.0134 (Asplund et al., 2009).

\section{Chemical and Dust Evolution Model of Galax- ies}

2.1 Equations of chemical and dust amount evolution

We deal with a galaxy composed of stars (including their remnants; i.e. white dwarfs, neutron stars, and black-holes) and the ISM. For simplicity, we assume the ISM to be one-zone. The ISM contains metal and dust as internal components. If we denote the masses of these components as $M_{*}$ (stars (and remnants)), $M_{\mathrm{ISM}}$ (ISM), $M_{Z}$ (metal), and $M_{\mathrm{d}}$ (dust), the equations describing their time evolutions are (e.g., Dwek, 1998)

$$
\begin{gathered}
\frac{d M_{*}}{d t}=S(t)-R(t) \\
\frac{d M_{\mathrm{ISM}}}{d t}=-S(t)+R(t)+I(t)-O(t) \\
\frac{d M_{Z}}{d t}=-Z(t) S(t)+Y_{Z}(t)+I_{Z}(t)-O_{Z}(t) \\
\frac{d M_{\mathrm{d}}}{d t}=-Z_{\mathrm{d}}(t) S(t)+Y_{\mathrm{d}}(t)-D_{\mathrm{SN}}(t)+G_{\mathrm{ac}}(t)+I_{\mathrm{d}}(t)-O_{\mathrm{d}}(t),
\end{gathered}
$$

where $S$ is the star-formation rate, $R$ is the mass-return rate from dying stars, $Y_{Z}$ and $Y_{\mathrm{d}}$ are the metal- and dustsupplying rate 'yields' by dying stars, respectively. $Z \equiv$ $M_{Z} / M_{\text {ISM }}$ is the metal-mass fraction in the ISM called 'metallicity', and $Z_{\mathrm{d}} \equiv M_{\mathrm{d}} / M_{\mathrm{ISM}}$ is the dust-mass fraction in the ISM which we call the dust-to-gas mass ratio. Note that $M_{\mathrm{ISM}}>M_{Z} \geq M_{\mathrm{d}}$.

$I, I_{Z}$, and $I_{\mathrm{d}}$ are the ISM, metal, and dust infall rates from the IGM, respectively. $O, O_{Z}$, and $O_{\mathrm{d}}$ are the ISM, metal, and dust outflow rates to the IGM, respectively. In this paper, we do not consider any outflows $\left(O=O_{Z}=\right.$ $O_{\mathrm{d}}=0$ ), but consider only an ISM infall $I$ (no metal and dust in the infalling gas: $I_{Z}=I_{\mathrm{d}}=0$ ), which is required to reproduce the metallicity distribution of stars nearby the Sun. ${ }^{1}$ The reason why we omit any outflows is that we do not know the transport mechanism of metal and dust from galaxies to the IGM (e.g., Bianchi and Ferrara, 2005). However, this omission may be inconsistent with detections of metal and dust in the IGM (e.g., Songaila and Cowie, 1996; Ménard et al., 2010). ${ }^{2}$

\footnotetext{
${ }^{1}$ Without gas infall from intergalactic space, we would expect a much larger number of low-metallicity stars in the solar neighborhood than is observed. This is called the 'G-dwarf problem' (e.g., Pagel, 1989).

${ }^{2}$ The origin of intergalactic metals and dust is galactic outflows and the amount ejected from galaxies is the same order of that remained in galaxies (e.g., Ménard et al., 2010 for dust; see also Inoue and Kamaya, 2003, 2004, 2010). Dust grains may be ejected from galaxies more efficiently than metals because the grains receive momentum through radiation pressure (Bianchi and Ferrara, 2005). Even in this case, our discussion about the dust-to-metal ratio in Section 5 would not be affected essentially by the omission of this selective removal of dust, although the set of model parameters which can reproduce the observations would change. In any case, this point would be interesting for future work.
} 
In the dust mass equation (Eq. (4)), there are two additional terms; $D_{\mathrm{SN}}$ is the dust-destruction rate by $\mathrm{SNe}$ and $G_{\mathrm{ac}}$ is the dust-growth rate in the ISM by metal accretion. These two terms are discussed in Section 2.5 and Section 2.6 in detail.

\subsection{Star formation and infall rates}

We adopt a simple recipe for star formation introduced by Schmidt (1959): $S \propto M_{\mathrm{ISM}^{p}}{ }^{p}$ (Schmidt law). The index $p$, called the Schmidt index, is observationally indicated to be $p=1-2$ (e.g., Kennicutt, 1998; Elmegreen, 2011) and some theoretical interpretations for the value have been presented (e.g., Dopita and Ryder, 1996). However, the value and its origin of the index is still an open problem (Elmegreen, 2011 and references therein). Fortunately, the choice of the index is not important, in fact, because in Section 4 we calibrate other model parameters so as to reproduce the observed star-formation history $S(t)$ in the solar neighborhood, which is essential. We here assume $p=1$ in order to solve the equations analytically in Section 5. In this case, we need a time-scale to give the starformation rate: star-formation time-scale, $\tau_{\mathrm{SF}}$ (see Table 1 in Section 4 for the values). Thus, the star-formation rate is given by

$$
S(t)=\frac{M_{\mathrm{ISM}}(t)}{\tau_{\mathrm{SF}}} .
$$

The infall from the IGM mimics the structure formation in the Universe based on the hierarchical scenario with cold dark-matter (e.g., Peacock, 1999); small galaxies are first formed at density peaks of the dark matter distribution in the Universe and they become larger and larger as they merge with each other and also obtain mass by an accretion process. Here, we simply assume a smooth exponential infall rate although the mass assembly of a galaxy is intrinsically episodic due to the merging process. This simplification is a kind of ensemble average of many galaxies and is appropriate when considering a mean property of galaxies. The infall rate which we adopt is

$$
I(t)=\frac{M_{\text {total }}}{\tau_{\text {in }}} \exp \left(-t / \tau_{\text {in }}\right),
$$

where $\tau_{\text {in }}$ is the infall time-scale and $M_{\text {total }}$ is the total mass which a galaxy obtains within the infinite time (see Table 1 in Section 4 for the values). Note that $M_{\text {total }}$ just gives the normalization of mass of a galaxy.

\subsection{Stellar mass spectrum and returned mass rate}

Salpeter (1955) first investigated the mass spectrum of stars in the solar neighborhood, corrected it for modulation by stellar evolution and death, and obtained the mass spectrum of stars when they are born, called the initial mass function (IMF) of stars. Salpeter's IMF is a power-law: $d N / d m=\phi(m) \propto m^{-q}$ with $q=2.35$. A lot of ensuring research, confirmed that the slope was quite universal, especially for massive stars, although there was a cut-off mass for low-mass stars (e.g., Kroupa, 2002; Chabrier, 2003 for reviews). We adopt here a simple functional form proposed by Larson (1998) which is essentially equivalent to the IMFs by Kroupa (2002) and Chabrier (2003) as

$$
\phi(m) \propto m^{-q} \exp \left(-m_{\mathrm{c}} / m\right),
$$

with a cut-off mass $m_{\mathrm{c}}$ and the range from $m_{\text {low }}$ to $m_{\text {up }}$. As a standard case, we adopt $p=2.35, m_{\mathrm{c}}=0.2 M_{\odot}$, $m_{\text {low }}=0.1 M_{\odot}$, and $m_{\text {up }}=100 M_{\odot}$. The cut-off mass well matches with the observed data compiled by Kroupa (2002). We normalize the IMF as $\int_{m_{\text {low }}}^{m_{\text {up }}} m \phi(m) d m=1$.

The mass-returned rate from dying stars, $R$, is given by

$$
R(t)=\int_{m_{\mathrm{lf}}(t)}^{m_{\mathrm{up}}}\left\{m-w\left(m, Z\left[t^{\prime}\right]\right)\right\} \phi(m) S\left(t^{\prime}\right) d m
$$

where

$$
t^{\prime}=t-\tau_{\mathrm{lf}}(m)
$$

is the time at which stars with mass $m$ dying at time $t$ are born, $\tau_{\mathrm{lf}}(m)$ is the stellar life-time, $w(m, Z)$ is the remnant mass of stars with mass $m$ and metallicity $Z$, and $m_{\mathrm{lf}}(t)$ is the minimum mass of stars dying at time $t$. This is the inverse function of $t=\tau_{\mathrm{lf}}(m)$. If time $t$ is less than the lifetime of the star with $m_{\text {up }}$, the returned rate $R=0$. We have assumed that the metallicity of a star is the same as the ISM metallicity at the time when the star is born.

The stellar life-time $\tau_{\mathrm{lf}}(m)$ is calculated by the formula of Raiteri et al. (1996) which is a fitting function of Padova stellar evolutionary tracks (Bertelli et al., 1994). This formula is a function of stellar mass $m$ and metallicity $Z$. However, the $Z$-dependence is weak. Thus, we neglect it (we always set $Z=Z_{\odot}$ in the formula).

\subsection{Stellar yields of 'metal' and dust}

When stars die, they eject a substantial mass of metal and dust into the ISM. The term driving the time evolution of metal mass given by Eq. (3) is the metal-supplying rate, $Y_{Z}$, called metal yield. Using the IMF, $\phi(m)$, and the starformation rate, $S(t)$, we can express the metal yield as

$$
Y_{Z}(t)=\int_{m_{\mathrm{lf}}(t)}^{m_{\mathrm{up}}} m_{Z}\left(m, Z\left[t^{\prime}\right]\right) \phi(m) S\left(t^{\prime}\right) d m,
$$

where $m_{Z}$ is the metal mass ejected from a star with mass $m$ and metallicity $Z$, and $t^{\prime}$ is given by Eq. (9).

The dust-supplying rate, $Y_{\mathrm{d}}$, called the dust yield can be expressed likewise:

$$
Y_{\mathrm{d}}(t)=\int_{m_{\mathrm{lf}}(t)}^{m_{\mathrm{up}}} m_{\mathrm{d}}\left(m, Z\left[t^{\prime}\right]\right) \phi(m) S\left(t^{\prime}\right) d m,
$$

where $m_{\mathrm{d}}$ is the dust mass ejected from a star with mass $m$ and metallicity $Z$, and $t^{\prime}$ is given by Eq. (9).

\subsection{Dust destruction by supernova blast waves}

Dust grains are destroyed by SN shock waves due to shattering and sputtering (e.g., Dwek and Arendt, 1992). This dust destruction is observed in some SNRs as described in Section 1. In this paper, we adopt the dust-destruction rate by SNe proposed by Dwek and Scalo (1980) and McKee (1989):

$$
D_{\mathrm{SN}}(t)=\frac{M_{\mathrm{d}}(t)}{\tau_{\mathrm{SN}}(t)}
$$

where the destruction time-scale $\tau_{\mathrm{SN}}$ is defined as the timescale during which all the ISM is swept by 'dust destructive' shock waves:

$$
\tau_{\mathrm{SN}}(t)=\frac{M_{\mathrm{ISM}}(t)}{\epsilon m_{\mathrm{SN}} R_{\mathrm{SN}}(t)}
$$


where $R_{\mathrm{SN}}$ is the $\mathrm{SN}$-occurrence rate, $m_{\mathrm{SN}}$ is the mass swept by a single $\mathrm{SN}$, and $\epsilon$ is the efficiency of the dust destruction. The $\mathrm{SN}$-occurrence rate is given by

$$
R_{\mathrm{SN}}(t)=\int_{8 M_{\odot}}^{40 M_{\odot}} \phi(m) S\left(t^{\prime}\right) d m
$$

where we have assumed the mass range for $\mathrm{SNe}$ to be $8-$ $40 M_{\odot}$ (Heger et al., 2003) and $t^{\prime}$ is given by Eq. (9). If $t<\tau_{\mathrm{lf}}\left(40 M_{\odot}\right), R_{\mathrm{SN}}=0$. Note that we consider only Type II SNe and neglect Type Ia SNe. The reason is discussed in Section 3.

The effective mass swept by a dust-destructive shock wave, $\epsilon m_{\mathrm{SN}}$ is the important parameter. It is estimated to be $\sim 1000 M_{\odot}$, namely $\epsilon \sim 0.1$ and $m_{\mathrm{SN}} \sim 10^{4}$ $M_{\odot}$ (McKee, 1989; Nozawa et al., 2006). Recent models for starburst galaxies in the early Universe often assume an effective mass of $\epsilon m_{\mathrm{SN}} \sim 100 M_{\odot}$ which is a factor of 10 smaller than our fiducial value (Dwek et al., 2007; Pipino et al., 2011; Gall et al., 2011a). Their argument is that starburst activity produces multiple $\mathrm{SNe}$ which make the ISM highly inhomogeneous and the dust-destruction efficiency decreases in such a medium. However, the solar neighborhood is not the case, and, thus, we keep $\epsilon m_{\mathrm{SN}} \sim$ $1000 M_{\odot}$.

\subsection{Dust growth by 'metal' accretion in the ISM}

In the ISM, atoms of some refractory elements (or refractory molecules) may accrete onto a dust grain and may become a part of the grain. We call this process the accretion growth of dust in the ISM (Draine, 1990). Note that this process does not need nucleation, and thus, can occur even in the ISM. A simple estimate of the growth rate is (e.g., Hirashita, 2000)

$$
G_{\mathrm{ac}}(t)=X_{\text {cold }} N_{\mathrm{d}}(t) \pi a^{2} s_{Z} v_{Z} \rho_{Z}^{\mathrm{gas}}(t),
$$

where $X_{\text {cold }} N_{\mathrm{d}}$ is the number of dust grains in cold dense clouds, $a$ is the grain radius, $s_{Z}$ is the sticking probability of accreting metals (atoms or molecules), $v_{Z}$ is the thermal velocity of the accreting metals and $\rho_{Z}^{\text {gas }}$ is the mass density of the accreting metals in the gas-phase. Note that all the quantities except for $X_{\text {cold }} N_{\mathrm{d}}$ in Eq. (15) are typical (or effective) values averaged over various grain radii, elements, and ISM phases. The gas-phase metal density is reduced to $\rho_{Z}^{\text {gas }}=\rho_{\text {ISM }}^{\text {eff }} Z(1-\delta)$, where $\rho_{\text {ISM }}^{\text {eff }}$ is an effective ISM mass density. We define it as a mass-weighted average density of various ISM phases, and, then, it is determined by the density of dense molecular clouds where the dust growth occurs. Note that $\delta=M_{\mathrm{d}} / M_{Z}$, the dust-to-metal mass ratio (the dust-depletion factor is $1-\delta$ ). For spherical grains, $N_{\mathrm{d}}=3 M_{\mathrm{d}} /\left(4 \pi a^{3} \sigma\right)$, where $\sigma$ is the typical material density of grains.

Equation (15) can be reduced to

$$
G_{\mathrm{ac}}(t)=\frac{M_{\mathrm{d}}(t)}{\tau_{\mathrm{ac}}(t)}
$$

The accretion growth time-scale $\tau_{\mathrm{ac}}$ is

$$
\tau_{\mathrm{ac}}(t)=\frac{\tau_{\mathrm{ac}, 0}}{Z(t)(1-\delta[t])},
$$

where the normalization $\tau_{\mathrm{ac}, 0}$ is the parameter determining the process:

$$
\tau_{\mathrm{ac}, 0}=\frac{4 a \sigma}{3 X_{\mathrm{cold}} s_{Z} v_{Z} \rho_{\mathrm{ISM}}^{\mathrm{eff}}} .
$$

This time-scale is very uncertain, but we will obtain $\tau_{\mathrm{ac}, 0}=3 \times 10^{6} \mathrm{yr}$ as the fiducial value in Section 4.2 in order to reproduce the dust-to-metal ratio in the solar neighborhood with an SN destruction efficiency of $\epsilon m_{\mathrm{SN}}=1000$ $M_{\odot}$. This value can be obtained with a set of parameters of $a=0.1 \mu \mathrm{m}$ (typical size in the ISM of the Milky Way), $\sigma=3 \mathrm{~g} \mathrm{~cm}^{-3}$ (compact silicates), $s_{Z}=1, v_{Z}=0.2 \mathrm{~km} \mathrm{~s}^{-1}$ $\left({ }^{56} \mathrm{Fe}\right.$ as an accreting metal atom and thermal temperature of $100 \mathrm{~K}), \rho_{\mathrm{ISM}}^{\text {eff }}=1 \times 10^{-22} \mathrm{~g} \mathrm{~cm}^{-3}$, and $X_{\text {cold }}=0.2$. This data set is just an example, but ensures that the time-scale is not outrageous.

There is a discussion that the lifetime of dense clouds (or the recycling time-scale of dense gas) should be longer than the accretion growth time-scale for an efficient dust growth (Zhukovska et al., 2008; Dwek and Cherchneff, 2011). According to these authors, the lifetime is long enough to realize an efficient dust growth in the Milky Way and even in starburst in the early Universe. Another issue is the effect of grain-size distribution which is discussed in Hirashita (2011).

\section{Stellar Remnant and 'Metal' and Dust Yields}

In this section, we present new simple formulas to describe the stellar remnant mass and yields of metal and dust which are useful to input into the chemical evolution codes. We represent all elements heavier than helium as just a 'metal' in the formulas for simplicity, while yields of various elements are presented in the literature. We consider three types of stellar death: white dwarfs through the AGB phase, core-collapse Type II SNe, and a direct collapse leading to a black-hole called a 'collapser' (Heger et $a l ., 2003)$. In this paper, we assume the mass range for the SNe to be $8-40 M_{\odot}$ (Heger et al., 2003). The stars with a mass below or above this mass range become AGB stars, or 'collapsers', respectively.

We neglect Type Ia SNe for simplicity. This population of $\mathrm{SNe}$ is the major source of the element iron (Iwamoto et al., 1999) and may be the source of iron dust (Calura et al., 2008). However, in respect of the total stardust mass budget, the contribution of $\mathrm{SNe}$ Ia relative to $\mathrm{SNe}$ II is always less than 1-10\% (Zhukovska et al., 2008; Pipino et al., 2011). Since we are dealing with metal and dust each as a single component, we can safely neglect the contribution of SNe Ia.

The remnant mass, $w(m, Z)$, is taken from model calculations of AGB stars (Karakas, 2010) and SNe (Nomoto et al., 2006). Figure 1 shows the remnant mass fraction relative to the initial stellar mass, $w / m$. This depends on the metallicity $Z$ because $Z$ in the stellar atmosphere determines the radiation pressure through opacity and the strength of the stellar wind in the course of the stellar evolution, and affects the remnant mass. However, as shown in Fig. 1, the dependence is weak, so we neglect it. We obtain 


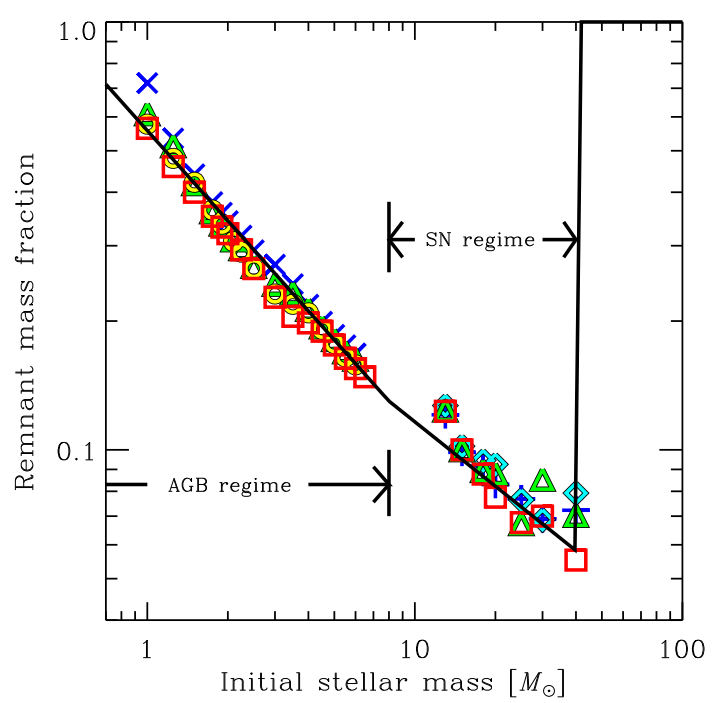

Fig. 1. Remnant mass fraction, $w / m$, as a function of the initial stellar mass, $m$. The data of AGB stars $\left(1-8 M_{\odot}\right)$ are taken from Karakas (2010) and those of $\mathrm{SNe}\left(8-40 M_{\odot}\right)$ are taken from Nomoto et al. (2006). The different symbols indicate a different metallicity $Z: Z=0$ (plus), $Z=0.0001$ (cross), $Z=0.001$ (diamond), $Z=0.004$ (triangle), $Z=0.008$ (circle), and $Z=0.02$ (square). The solid line is a fitting function given by Eq. (19).

the following fitting formula:

$$
\frac{w(m)}{m}=\left\{\begin{array}{ll}
1 & \left(m>40 M_{\odot}\right) \\
0.13\left(\frac{m}{8 M_{\odot}}\right)^{-0.5} & \left(8 M_{\odot} \leq m \leq 40 M_{\odot}\right) \\
0.13\left(\frac{m}{8 M_{\odot}}\right)^{-0.7} & \left(m<8 M_{\odot}\right)
\end{array},\right.
$$

which is shown by the solid line in Fig. 1. This fitting formula agrees with the values in table 1 of Morgan and Edmunds (2003) within a $<20 \%$ difference, except for the case $m=9 M_{\odot}$ for which our estimate is a factor of 2 lower than that of Morgan and Edmunds (2003).

For the metal yield, $m_{Z}$, we adopt the data taken from model calculations of AGBs (Karakas, 2010) and SNe (Nomoto et al., 2006). Figure 2 shows $m_{Z}$ relative to the initial stellar mass $m$ as a function of $m$. While the expected $m_{Z}$ depends on the mass $m$ and metallicity $Z$ in a complex way, we approximate the data with a simple power-law of only $m$ as

$$
\frac{m_{Z}(m)}{m}= \begin{cases}0 & \left(m>40 M_{\odot}\right) \\ f_{Z}\left(\frac{m}{8 M_{\odot}}\right)^{2} & \left(8 M_{\odot} \leq m \leq 40 M_{\odot}\right) \\ f_{Z}\left(\frac{m}{8 M_{\odot}}\right)^{0.7} & \left(m<8 M_{\odot}\right)\end{cases}
$$

When the normalization $f_{Z}=0.02$, Eq. (20) is the solid line in Fig. 2. As shown in the figure, the uncertainty of Eq. (20) is a factor of $\sim 2$. This fitting agrees with the values in table 1 of Morgan and Edmunds (2003) within a factor of 2 difference in the $\mathrm{SN}$ regime. However, in the AGB regime, the difference is as large as the model results by Karakas (2010). The effect of this large uncertainty in the yield is discussed in Section 6.1.

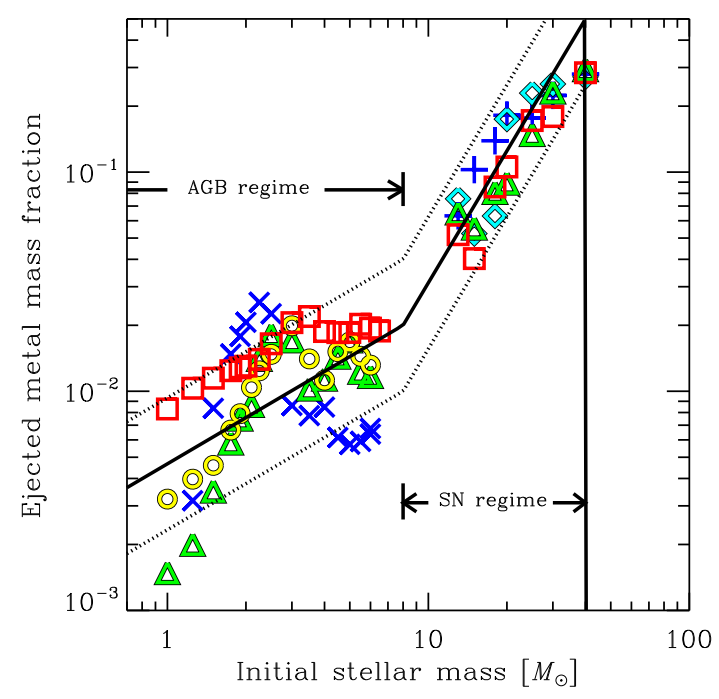

Fig. 2. Ejected metal mass fraction, $m_{Z} / m$, as a function of the initial stellar mass, $m$. The data of AGB stars $\left(1-8 M_{\odot}\right)$ are taken from Karakas $(2010)$ and those of SNe $\left(8-40 M_{\odot}\right)$ are taken from Nomoto et al. (2006). The meaning of the symbols are the same as in Fig. 1. The solid line is a fitting function given by Eq. (20). The dotted lines are the cases a factor of two higher or lower than the solid line.

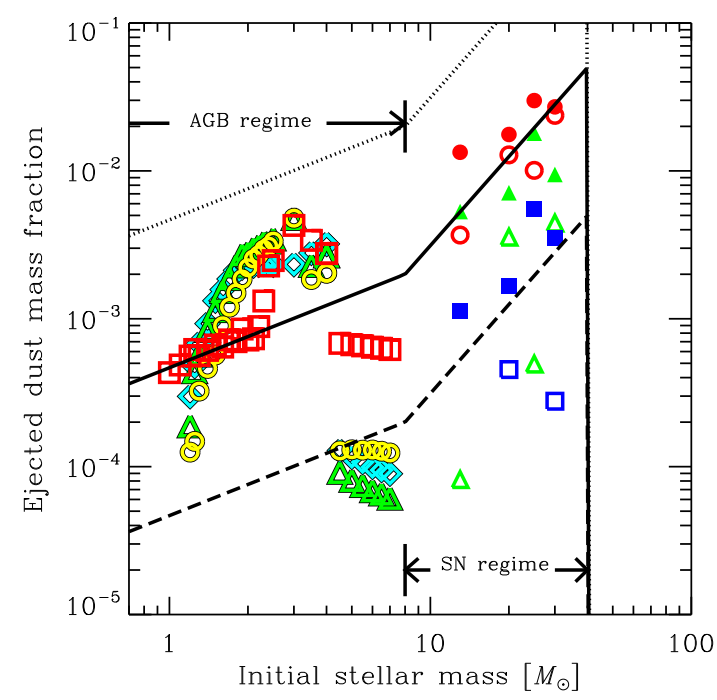

Fig. 3. Ejected dust mass fraction, $m_{\mathrm{d}} / m$, as a function of the initial stellar mass, $m$. The data of AGB stars $\left(1-8 M_{\odot}\right)$ are taken from Zhukovska et al. (2008) and those of SNe $\left(8-40 M_{\odot}\right)$ are taken from Nozawa et al. (2007). For the AGB data, the different symbols indicate a different metallicity $Z: Z=0.001$ (diamond), $Z=0.004$ (triangle), $Z=0.008$ (circle), and $Z=0.02$ (square). For the $\mathrm{SNe}$ data, the different symbols indicate a different ambient hydrogen density $n_{\mathrm{H}}=0.1 \mathrm{~cm}^{-3}$ (circle), $n_{\mathrm{H}}=1 \mathrm{~cm}^{-3}$ (triangle), and $n_{\mathrm{H}}=10 \mathrm{~cm}^{-3}$ (square). The open and filled symbols correspond to 'mixed' or 'unmixed' cases of Nozawa et al. (2007), respectively. The dotted, solid, and dashed lines are the cases of $\xi=1,0.1$, and 0.001 , respectively, in Eq. (21).

The dust yield, $m_{\mathrm{d}}$, calculated by Zhukovska et al. (2008) and Ferrarotti and Gail (2006) for AGBs and Nozawa et al. (2007) for SNe are shown in Fig. 3. These yields are theoretical ones and have not been compared with observations very much yet. As found in Fig. 3, $m_{\mathrm{d}}$ depends on the mass $m$ and metallicity $Z$ in a complex way, as does the metal yield $m_{Z}$. Moreover, the dust production by $\mathrm{SNe}$ is further complex because the reverse shock moving in the ejecta of 
Table 1. Parameters and values for the solar neighborhood.

\begin{tabular}{lll}
\hline Parameter & Fiducial value & Considered values \\
\hline$\left(\tau_{\mathrm{SF}} / \mathrm{Gyr}, \tau_{\mathrm{in}} / \mathrm{Gyr}\right)$ & $(3,15)$ & $(1,50),(2,20),(3,15)$, and $(5,10)$ \\
\hline$\left(\tau_{\mathrm{ac}, 0} / \mathrm{Myr}, \epsilon m_{\mathrm{SN}} / 10^{3} M_{\odot}\right)$ & $(3,1)$ & $(1.5,1),(1.5,2),(3,0.5),(3,1),(3,2),(6,0.5)$, and $(6,1)$ \\
\hline$f_{Z}$ & 0.02 & $0.01,0.02$, and 0.04 \\
$\xi$ & 0.1 & $0.01,0.1$, and 1 \\
\hline
\end{tabular}

an SN may destroy the dust produced in the ejecta (Bianchi and Schneider, 2007; Nozawa et al., 2007; Nath et al., 2008; Silvia et al., 2010). This self-destruction depends on the material strength opposing the destruction ${ }^{3}$ and the ambient gas density which determines the strength of the reverse shock. According to Nozawa et al. (2007), we plot three cases of the ambient density and 'mixed' and 'unmixed' dust productions ${ }^{4}$ in Fig. 3. We adopt a simple formula for $m_{\mathrm{d}}$ as

$$
m_{\mathrm{d}}(m)=\xi m_{Z}(m)
$$

where $\xi$ is a scaling factor and indicates the efficiency of condensation of metal elements. In Fig. 3, we show three cases of $\xi=1$ (all metal condenses into dust: an extreme but unrealistic case), 0.1 (fiducial case), and 0.01 (a lower efficiency case). The reader may be anxious about the large uncertainty of this approximation. However, the dust mass in galaxies does not depend on $m_{\mathrm{d}}$ after accretion growth becomes active. This is because the growth of dust is the dominant process of dust production after the activation as shown later in Section 6.1.

\section{Milky Way Analog}

Let us calibrate the parameters in the chemical and dust evolution model of galaxies so as to reproduce the properties of the solar neighborhood in the Milky Way. There are two parameters in the chemical evolution part: the timescales of star formation, $\tau_{\mathrm{SF}}$, and infall, $\tau_{\text {in }}$. There are two additional parameters in the dust-content evolution: the time-scale of the ISM accretion growth, $\tau_{\mathrm{ac}, 0}$, and the efficiency of the dust destruction, $\epsilon m_{\mathrm{SN}}$. In addition, there are two parameters reflecting the uncertainties of the metal and dust yields, $f_{Z}$ and $\xi$. Table 1 is a summary of these parameters and values.

Note that we do not apply any statistical method to justify the goodness of the reproduction of the observational constraints throughout this paper because our aim is not to find the best fit solution for the constraints but to demonstrate the dust-content evolution in galaxies qualitatively. This is partly due to the weakness of the observational constraints and due to the large uncertainties of dust physics itself.

\subsection{Chemical evolution at the solar neighborhood}

Here, we determine the time-scales of star-formation and infall in the chemical evolution part. First, we constrain these time-scales by using the star-formation history at the

\footnotetext{
${ }^{3}$ The micro-process of the destruction considered in Nozawa et al. (2007) is sputtering by hot gas.

${ }^{4}$ The terms 'mixed' and 'unmixed' refer to the elemental mixing in the SN ejecta (Nozawa et al., 2003). In the 'mixed' case, there is no layer where $\mathrm{C}$ is more abundant than $\mathrm{O}$, so only silicate, troilite, and corundum grains can be formed. On the other hand, the 'unmixed' case has a C-rich layer and $\mathrm{Fe}$ layer and can form carbon and iron grains as well as silicate.
}

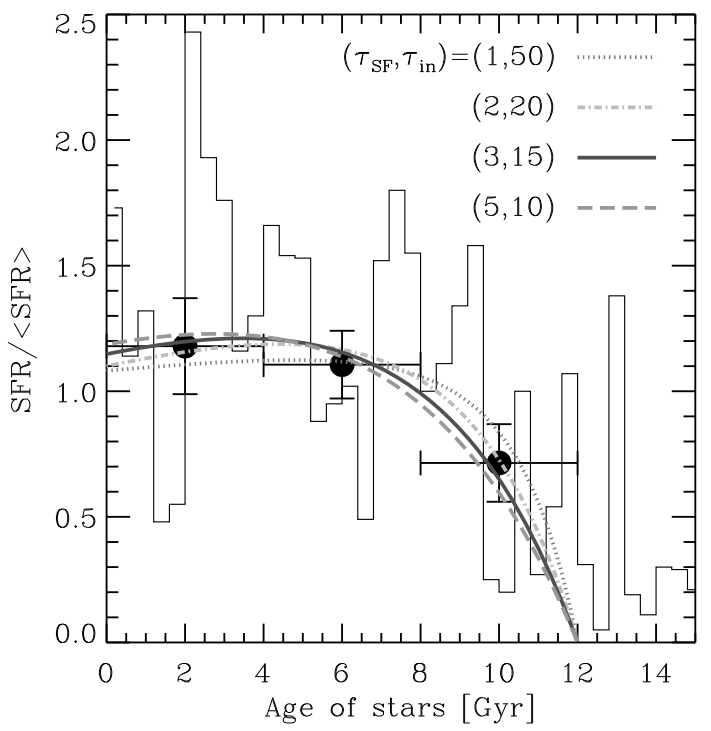

Fig. 4. Star-formation history (time evolution of star formation rate) normalized by the average rate. The histogram is the observed history at the solar neighborhood reported by Rocha-Pinto et al. (2000a). The filled circles with error-bars are the average of the histogram over a 4-Gyr interval and corrected by the average rate only for an age less than 12 Gyr which is the present age of the Milky Way assumed in this paper. The vertical error-bars are the standard error of the mean. The four lines correspond to the time evolutions with four different sets of time-scales of star formation and infall $\left(\tau_{\mathrm{SF}} / \mathrm{Gyr}, \tau_{\mathrm{in}} / \mathrm{Gyr}\right)$ as indicated in the panel.

solar neighborhood reported by Rocha-Pinto et al. (2000a). Such a method was adopted by Takeuchi and Hirashita (2000). Rocha-Pinto et al. (2000a) derived the star formation history from the age distribution of 552 late-type dwarf stars at the solar neighborhood. The histogram in Fig. 4 is their result and shows the very stochastic nature of the history. However, our model can treat only a smooth history. Thus, we have smoothed the stochastic history by averaging with a 4-Gyr interval. The filled circles are the result. The vertical error-bars indicate the standard error of the mean. The average history is re-normalized by the average starformation rate for a stellar age less than $12 \mathrm{Gyr}$, which is the assumed age of the Milky Way in this paper, although this choice of the age is arbitrary. We have tried four cases of $\tau_{\mathrm{SF}}$ in this paper: $1,2,3$, and $5 \mathrm{Gyr}$ which are the observed range of the time-scale (or gas consumption timescale) for disk galaxies like the Milky Way (e.g., Larson et al., 1980). For each $\tau_{\mathrm{SF}}$, we have found $\tau_{\text {in }}$ with which we can reproduce the smoothed history as shown in Fig. 4.

Next, we adopt the observed relation between the stellar age and metallicity, the so-called age-metallicity relation, reported by Rocha-Pinto et al. (2000b), to further constrain $\left(\tau_{\mathrm{SF}}, \tau_{\text {in }}\right)$. Rocha-Pinto et al. (2000b) derived the relation 


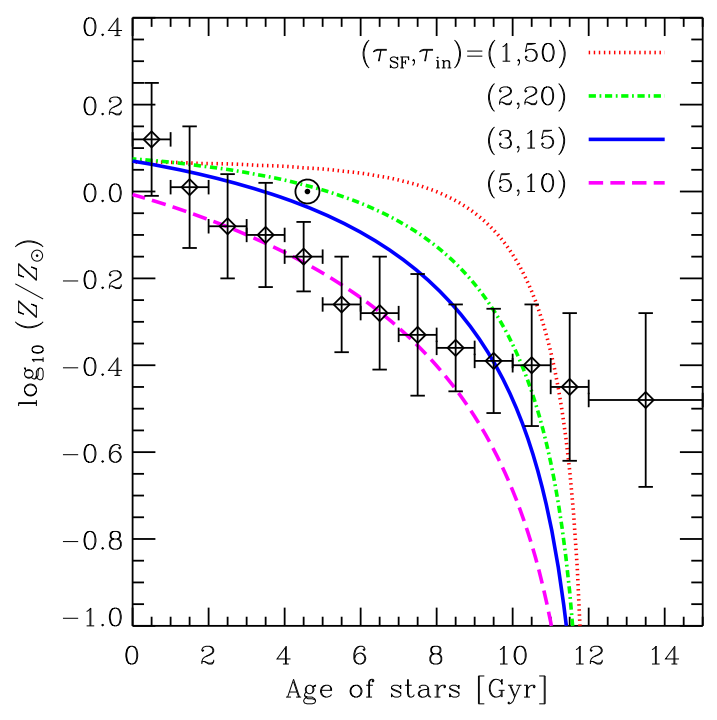

Fig. 5. Age-metallicity relation of stars. The diamonds with error-bars are the data of stars at the solar neighborhood reported by Rocha-Pinto et al. (2000b). The solar mark $(\odot)$ indicates the position of the Sun on this plot. The four lines correspond to the model relations the same as Fig. 4. The present age of the Milky Way is assumed to be $12 \mathrm{Gyr}$.

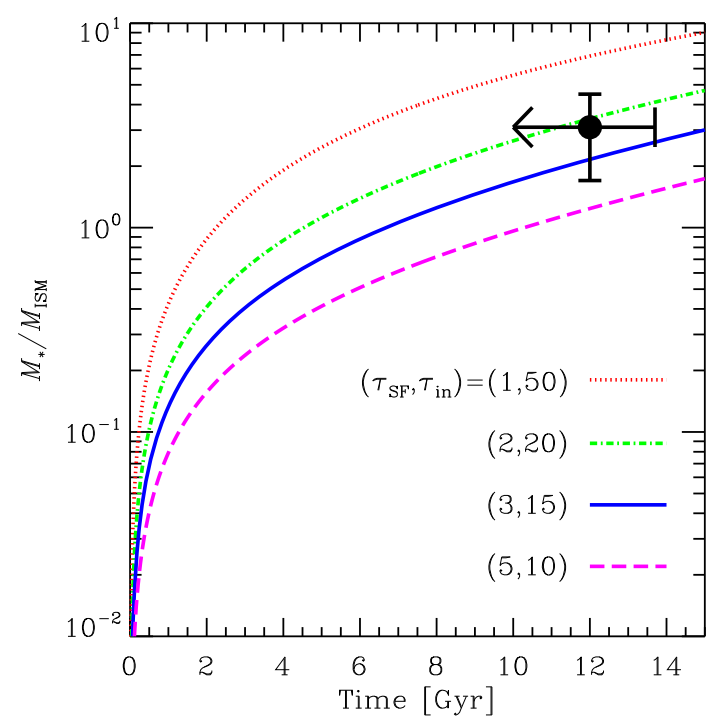

Fig. 6. Time evolution of the stellar mass relative to the ISM mass. The filled circle with error-bars is an estimate in the solar neighborhood, taken from Naab and Ostriker (2006) and references therein. Note that the stellar mass includes the mass of remnants. The present age of the Milky Way should be less than the age of the Universe (13.7 Gyr). The four lines correspond to the model evolutions as in Fig. 4.

from the same 552 stars as Rocha-Pinto et al. (2000a). Their result is shown in Fig. 5 by diamonds with error-bars. After comparing their result with our four model lines, we find that the $\left(\tau_{\mathrm{SF}} / \mathrm{Gyr}, \tau_{\mathrm{in}} / \mathrm{Gyr}\right)=(5,10)$ case seems the best match with the observed relation but $\left(\tau_{\mathrm{SF}} / \mathrm{Gyr}, \tau_{\text {in }} / \mathrm{Gyr}\right)=$ $(3,15)$ case is also acceptable.

Finally, we adopt another constraint: the current stellar mass relative to the ISM mass. Naab and Ostriker (2006) compiled observational constraints for the solar neighborhood. From the compilation, we adopt the ratio of the stellar mass to the ISM mass at the present epoch of 3.1 \pm 1.4 . Note that the stellar mass includes the remnant mass (i.e. white dwarfs, neutron stars, and black-holes). Figure 6 shows the comparison of the ratio with our four star-formation histories. We have found that the two sets of $\left(\tau_{\mathrm{SF}} / \mathrm{Gyr}, \tau_{\mathrm{in}} / \mathrm{Gyr}\right)=(2,20)$ and $(3,15)$ are consistent with the data.

From these three comparisons, we finally adopt the case of $\left(\tau_{\mathrm{SF}} / \mathrm{Gyr}, \tau_{\mathrm{in}} / \mathrm{Gyr}\right)=(3,15)$ as the fiducial set for the Milky Way (or more precisely, for the solar neighborhood) in this paper.

\subsection{Dust content evolution at the solar neighborhood}

Here we examine the dust-content evolution. First, we show the significant effect of dust destruction and ISM growth. Figure 7 shows the time evolution of metallicity and dust-to-gas mass ratio for the fiducial set of $\tau_{\mathrm{SF}}$ and $\tau_{\text {in }}$ obtained in the previous subsection. The model curves of the dust-to-gas ratio (dotted, dot-dashed, and solid lines) are compared with the filled circle with error-bars which is an observational estimate for the solar neighborhood. This is obtained from a metallicity $Z \approx Z_{\odot}$ (van den Bergh, 2000; see also Rocha-Pinto et al., 2000b) and dust-to-metal mass ratio $\delta \approx 0.5$ (Kimura et al., 2003; see below) and the uncertainty is the quadrature of uncertainties of $30 \%$ in $Z$ and $20 \%$ in $\delta$.

If there is neither destruction nor accretion growth of dust, the dust-to-gas ratio evolution is just the metallicity evolution multiplied by the condensation efficiency of stardust, $\xi$, as shown by the dotted line. We have assumed $\xi=0.1$ for this line. Once the SN destruction of dust is turned on with a standard efficiency as $\epsilon m_{\mathrm{SN}}=1 \times 10^{3} M_{\odot}$

$\overline{5}^{5}$ The difference between the $Z_{\odot}$ values of Anders and Grevesse (1989) and Asplund et al. (2009) accounts for the uncertainty.

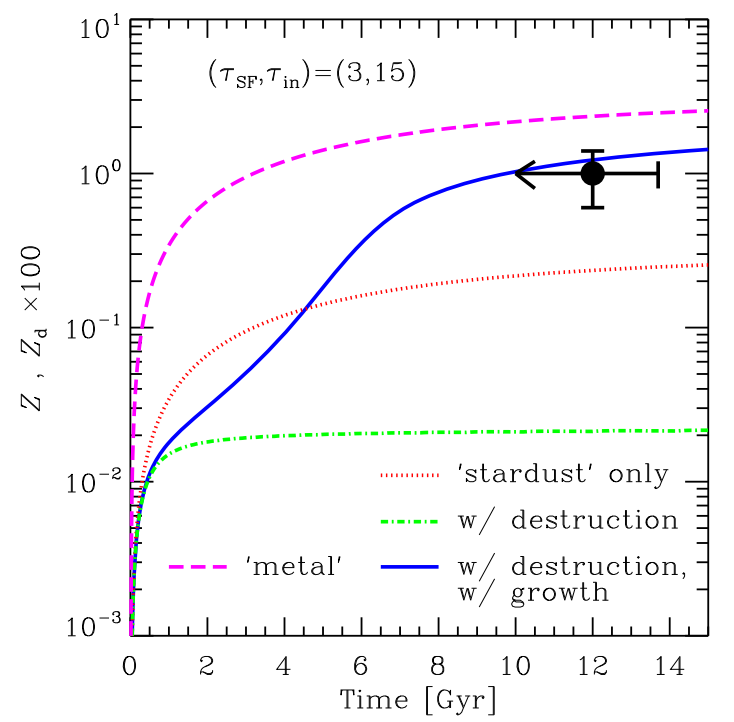

Fig. 7. Time evolution of metallicity (metal mass fraction in the ISM; dashed line) and dust-to-gas mass ratio (other lines) for the fiducial case (Table 1). The dotted line corresponds to the case only with 'stardust' production and destruction by star formation (i.e. astration). The dot-dashed line corresponds to the case with dust destruction by $\mathrm{SNe}$ but without dust growth in the ISM. The solid line is the case with all the processes. The filled circle with error-bars is an estimate of the dust-to-gas mass ratio for the solar neighborhood (see text). The present age of the Milky Way should be less than the age of the Universe (13.7 Gyr). 


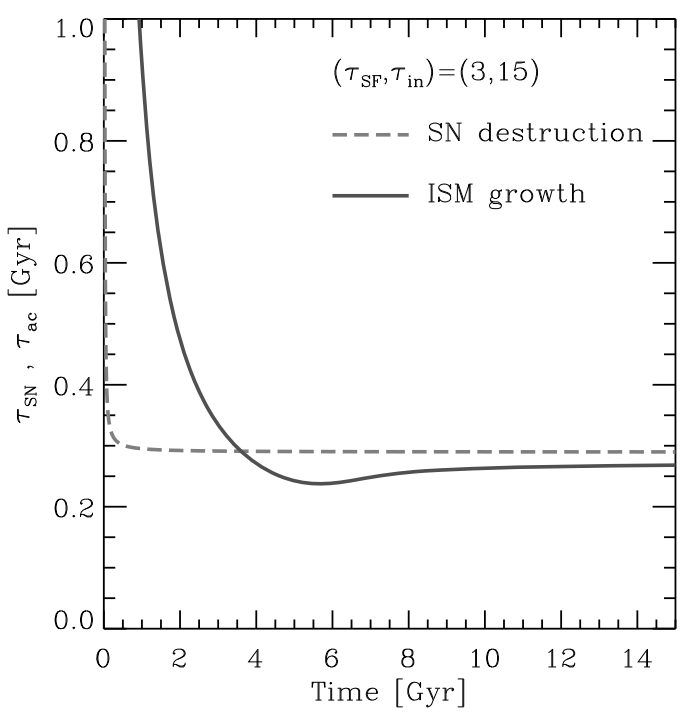

Fig. 8. Time evolution of time-scales of the dust destruction by $\mathrm{SNe}$ (dashed line) and of the dust growth in the ISM (solid line) for the fiducial case (Table 1).

(McKee, 1989; Nozawa et al., 2006), the dust amount is reduced by a factor of ten as shown by the dot-dashed line. This confirms that the dust destruction is very efficient and the stardust injection is too small to compensate for the destruction (e.g., Draine, 1990; Tielens, 1998). Then we need the accretion growth in the ISM to reproduce the dust-togas ratio, $\sim 10^{-2}$, in the present Milky Way. If we assume a time-scale of $\tau_{\mathrm{ac}, 0}=3 \times 10^{6} \mathrm{yr}$, the dust-to-gas-ratio evolution becomes the solid line and reaches $\simeq 10^{-2}$ which, after several Gyr, is almost two orders of magnitude larger than the case without growth.

Figure 8 shows the time evolution of $\tau_{\mathrm{SN}}$ in Eq. (13) and $\tau_{\mathrm{ac}}$ in Eq. (17). The $\mathrm{SN}$ destruction time-scale $\tau_{\mathrm{SN}}$ is almost constant promptly after the first few hundreds of Myr. On the other hand, the accretion growth time-scale $\tau_{\mathrm{ac}}$ decreases gradually in the first few Gyr. This is because $\tau_{\mathrm{ac}}$ has a metallicity dependence as shown in Eq. (17) and decreases as the metallicity increases. At a time around $4 \mathrm{Gyr}$, $\tau_{\mathrm{ac}}$ becomes shorter than $\tau_{\mathrm{SN}}$, and then the accretion growth becomes significant and the dust amount increases rapidly. As the accretion growth proceeds, the metal abundance in the gas phase decreases, i.e. the dust-to-metal ratio $\delta$ increases, then $\tau_{\mathrm{ac}}$ becomes almost constant and balances with $\tau_{\mathrm{SN}}$. We will discuss this point in Section 5 in more detail.

Figure 9 shows the time evolution of the dust-to-metal ratio, $\delta$. The solid line is the fiducial case which is shown in Figs. 7 and 8. This can be compared with the observed ratio in the Local Interstellar Cloud reported by Kimura et al. (2003): $\delta=0.5 \pm 0.1$. As shown in Fig. 9, the fiducial set of $\left(\tau_{\mathrm{ac}, 0}, \epsilon m_{\mathrm{SN}}\right)=\left(3 \mathrm{Myr}, 1 \times 10^{3} M_{\odot}\right)$ agrees excellently with the observed data. On the other hand, other sets can also reproduce the data. For example, $\left(\tau_{\mathrm{ac}, 0}, \epsilon m_{\mathrm{SN}}\right)=$ $\left(1.5 \mathrm{Myr}, 2 \times 10^{3} M_{\odot}\right)$ or $\left(6 \mathrm{Myr}, 5 \times 10^{2} M_{\odot}\right)$. Interestingly, the $\delta$ evolutions become very similar if the product of $\tau_{\mathrm{ac}, 0}$ and $\epsilon m_{\mathrm{SN}}$ is the same. We will also discuss this point in Section 5.

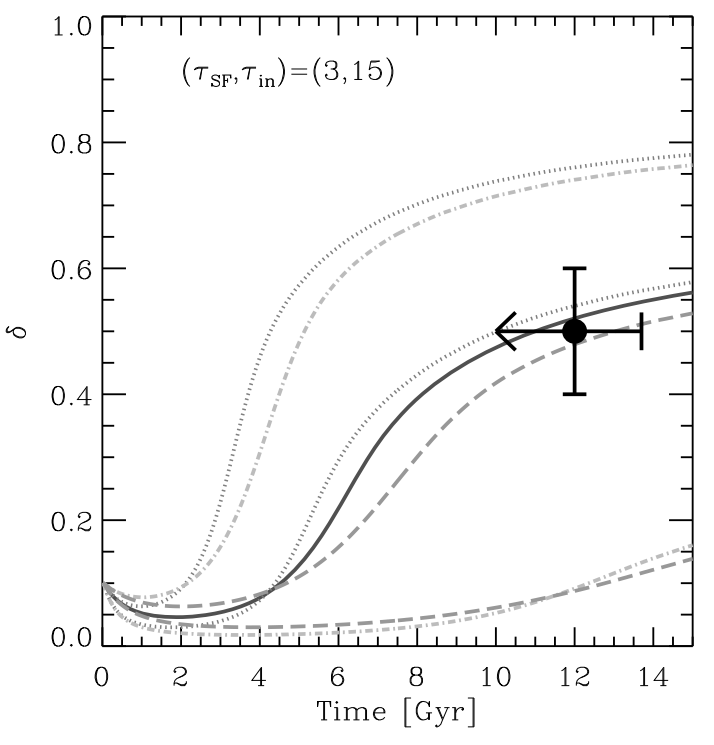

Fig. 9. Time evolution of dust-to-metal mass ratio, $\delta$, for various sets of the parameters of dust growth and destruction $\left(\tau_{\mathrm{ac}, 0}, \epsilon m_{\mathrm{SN}}\right)$. The solid line is the fiducial case with $\left(3 \times 10^{6} \mathrm{yr}, 1 \times 10^{3} M_{\odot}\right)$. The two dotted lines correspond to the two different sets of $\left(1.5 \times 10^{6} \mathrm{yr}, 1 \times 10^{3} M_{\odot}\right)$ for the upper and $\left(1.5 \times 10^{6} \mathrm{yr}, 2 \times 10^{3} M_{\odot}\right)$ for the lower. The two dot-dashed lines correspond to the sets of $\left(3 \times 10^{6} \mathrm{yr}, 5 \times 10^{2} M_{\odot}\right)$ for the upper and $\left(3 \times 10^{6} \mathrm{yr}, 2 \times 10^{3} M_{\odot}\right)$ for the lower. The two dashed lines correspond to the sets of $\left(6 \times 10^{6} \mathrm{yr}, 5 \times 10^{2} M_{\odot}\right)$ for the upper and $\left(6 \times 10^{6} \mathrm{yr}, 1 \times 10^{3} M_{\odot}\right)$ for the lower. The filled circle with error-bars is an estimate in the Local Interstellar Cloud surrounding the Sun by Kimura et al. (2003). The present age of the Milky Way should be less than the age of the Universe (13.7 Gyr).

\section{Determining Dust-to-Metal Ratio}

In this section, we demonstrate the mechanism for determining the dust-to-metal mass ratio, $\delta$, in galaxies. Starting from Eqs. (3) and (4), we can obtain the time-evolutionary equation of $\delta \equiv M_{\mathrm{d}} / M_{Z}$. Here, let us adopt the instantaneous recycling approximation (IRA) in which we neglect the finite stellar life-time and assume that stars with a mass larger than a certain threshold (the turn-off mass $m_{\mathrm{t}}$ ) die instantly when they are formed. This approximation allows us to manage the equations analytically and is good enough to see phenomena with a time-scale longer than a Gyr (see Tinsley, 1980 for more details). In the IRA, we can approximate the metal and dust yields in Eqs. (10) and (11) as $Y_{Z} \approx \mathcal{Y}_{Z} S$ and $Y_{\mathrm{d}}=\xi Y_{Z} \approx \xi \mathcal{Y}_{Z} S$, where the effective metal yield

$$
\mathcal{Y}_{Z}=\int_{m_{\mathrm{t}}}^{m_{\mathrm{up}}} m_{Z}(m) \phi(m) d m=0.024\left(\frac{f_{Z}}{0.02}\right),
$$

where we have assumed $m_{\mathrm{t}}=1 M_{\odot}$. This value is not sensitive to $m_{\mathrm{t}}$. We obtain $\mathcal{Y}_{Z}=0.021\left(f_{Z} / 0.02\right)$ if $m_{\mathrm{t}}=5$ $M_{\odot}$. Remembering the star-formation rate $S=M_{\mathrm{ISM}} / \tau_{\mathrm{SF}}$ as in Eq. (5), then, we obtain

$$
\frac{1}{\delta} \frac{d \delta}{d t} \approx-\frac{\mathcal{Y}_{Z}}{\tau_{\mathrm{SF}} Z}\left(1-\frac{\xi}{\delta}\right)-\frac{1}{\tau_{\mathrm{SN}}}+\frac{1}{\tau_{\mathrm{ac}}} .
$$

In the IRA, the $\mathrm{SN}$ destruction time-scale $\tau_{\mathrm{SN}}$ in Eq. (13) can be reduced to

$$
\tau_{\mathrm{SN}} \approx \frac{\tau_{\mathrm{SF}}}{\epsilon m_{\mathrm{SN}} n_{\mathrm{SN}}}
$$


where the effective number of SN per unit stellar mass is

$$
n_{\mathrm{SN}}=\int_{8 M_{\odot}}^{40 M_{\odot}} \phi(m) d m=0.010 M_{\odot}^{-1} .
$$

Note that $\epsilon m_{\mathrm{SN}} n_{\mathrm{SN}}$ is a non-dimensional value. The accretion growth time-scale $\tau_{\mathrm{ac}}$ is given in Eq. (17). Then, Eq. (23) is reduced to

$$
\frac{1}{\delta} \frac{d \delta}{d t} \approx-\frac{\alpha+\epsilon m_{\mathrm{SN}} n_{\mathrm{SN}}}{\tau_{\mathrm{SF}}}+\frac{Z(1-\delta)}{\tau_{\mathrm{ac}, 0}}
$$

where

$$
\alpha=\frac{\mathcal{Y}_{Z}}{Z}\left(1-\frac{\xi}{\delta}\right) .
$$

In the IRA, the metallicity $Z \equiv M_{Z} / M_{\text {ISM }}$ can be obtained analytically (for example, see Dwek et al., 2007). Then, we have found that $Z \rightarrow \mathcal{Y}_{Z}$ for $t \rightarrow \infty$ when $\tau_{\text {in }}>\tau_{\text {SF. }}$. The condensation efficiency $\xi$ is uncertain but is of the order of 0.1 (see Fig. 3). When $\delta$ is of the order of $0.1-1$ as shown in Fig. 9, the ratio $\xi / \delta$ is of the order of 1 or smaller. Therefore, $\alpha$ is also of the order of 1 or smaller. On the other hand, $n_{\mathrm{SN}} \sim 10^{-2} M_{\odot}^{-1}$ and $\epsilon m_{\mathrm{SN}} \sim 10^{3} M_{\odot}$, then we obtain $\epsilon m_{\mathrm{SN}} n_{\mathrm{SN}} \gg \alpha$. Therefore, Eq. (26) is further reduced to

$$
\frac{1}{\delta} \frac{d \delta}{d t} \approx-a+b(1-\delta),
$$

where $a=\epsilon m_{\mathrm{SN}} n_{\mathrm{SN}} / \tau_{\mathrm{SF}}$ and $b=Z / \tau_{\mathrm{ac}, 0}$. If we assume $Z$ to be constant (i.e. $b$ is constant), Eq. (28) can be solved analytically. The solution is

$$
\delta \approx \frac{\delta_{\infty} \delta_{0} \exp (b-a) t}{\left(\delta_{\infty}-\delta_{0}\right)+\delta_{0} \exp (b-a) t},
$$

where $\delta_{0}$ and $\delta_{\infty}$ are the values for $t=0$ and $t \rightarrow \infty$, respectively. The asymptotic value $\delta_{\infty}$ for $t \rightarrow \infty$ is realized only when $b>a$, and is given by

$$
1-\delta_{\infty}=\frac{a}{b}=\frac{\tau_{\mathrm{ac}, 0} \in m_{\mathrm{SN}} n_{\mathrm{SN}}}{\tau_{\mathrm{SF}} Z} .
$$

This is the equilibrium value for Eq. (28) and we find

$$
\begin{array}{r}
1-\delta_{\infty}=0.5\left(\frac{\tau_{\mathrm{ac}, 0}}{3 \mathrm{Myr}}\right)\left(\frac{\epsilon m_{\mathrm{SN}}}{10^{3} M_{\odot}}\right)\left(\frac{n_{\mathrm{SN}}}{10^{-2} M_{\odot}^{-1}}\right) \\
\times\left(\frac{3 \mathrm{Gyr}}{\tau_{\mathrm{SF}}}\right)\left(\frac{0.02}{Z}\right),
\end{array}
$$

which excellently agrees with the results in Fig. 9.

We can fully understand the $\delta$ evolution by using Eq. (28). At the beginning, the accretion term $b \sim 0$ because $Z \sim 0$. Then, only the destruction term $a$ is effective. As a result, $\delta$ decreases with the time-scale of $1 / a=\tau_{\mathrm{SN}}$. As $Z$ increases, the accretion term $b$ increases and finally exceeds $a$. Then, $\delta$ increases toward $\delta_{\infty}$ with the evolution time-scale of $1 /(b-a)$. This decreases as $Z$ increases and $b-a$ increases. Therefore, the driving force of the $\delta$ evolution is $Z$. If we call $Z$ at $b=a$ the critical metallicity, $Z_{\mathrm{c}}$, we find

$$
Z_{\mathrm{c}}=\frac{\tau_{\mathrm{ac}, 0} \epsilon m_{\mathrm{SN}} n_{\mathrm{SN}}}{\tau_{\mathrm{SF}}}=0.01
$$

$$
\times\left(\frac{\tau_{\mathrm{ac}, 0}}{3 \mathrm{Myr}}\right)\left(\frac{\epsilon m_{\mathrm{SN}}}{10^{3} M_{\odot}}\right)\left(\frac{n_{\mathrm{SN}}}{10^{-2} M_{\odot}^{-1}}\right)\left(\frac{3 \mathrm{Gyr}}{\tau_{\mathrm{SF}}}\right) .
$$

When $Z>Z_{\mathrm{c}}$, the accretion growth becomes effective and $\delta$ approaches the final value $\delta_{\infty}$. A similar critical metallicity has been derived by Asano et al. (2011) in a different way.

Equation (30) shows that the final value of $\delta$ is determined by the equilibrium between the $\mathrm{SN}$ destruction and the accretion growth in the ISM. The time-scale to reach equilibrium is $1 /(b-a)$. This is relatively short in the fiducial case. For example, it is $0.3 \mathrm{Gyr}$ when $Z=0.02$. This means that the $\delta$ evolution proceeds with keeping the equilibrium between $\mathrm{SN}$ destruction and the accretion growth, or equivalently, $\delta=\delta_{\infty}$ after $Z$ exceeds $Z_{\mathrm{c}}$. This behavior is also found by comparing the two time-scales, $\tau_{\mathrm{SN}}$ and $\tau_{\mathrm{ac}}$ in Fig. 8; once $\tau_{\mathrm{ac}}$ becomes shorter than $\tau_{\mathrm{SN}}$ at about $4 \mathrm{Gyr}$ at which $Z$ exceeds $Z_{\mathrm{c}}, \tau_{\mathrm{ac}}$ turns around and approaches $\tau_{\mathrm{SN}}$ again. This is realized by the reduction of the term $(1-\delta)$ in $\tau_{\text {ac }}$ (see Eq. (17)) when $\delta$ increases from $\sim 0$ to $\delta_{\infty}$. Such a kind of self-regulation process determines the dust-to-metal ratio $\delta$.

\section{Discussion}

\subsection{Effect of uncertainties of yields}

Here, we examine the effect of uncertainties of the normalization of metal and dust yields. As we saw in Fig. 2, our simple recipe for the metal yield may contain a factor of 2 (or more) uncertainty. The parameter $f_{Z}$ accounts for this uncertainty. In Fig. 10, we show the effect of $f_{Z}$. As found from the panel (a), the metallicity evolution is scaled almost linearly by $f_{Z}$ as expected and the timing at which $Z$ exceeds $Z_{\mathrm{c}}=0.5 Z_{\odot}$ given by Eq. (32) for the fiducial set of the accretion and destruction efficiencies becomes faster as $f_{Z}$ is larger. From the panels (b) and (c), we find that for each case of $f_{Z}, \delta$ increases and $\tau_{\mathrm{ac}}$ becomes shorter than $\tau_{\mathrm{SN}}$ soon after the timing for $Z>Z_{\mathrm{c}}$. Therefore, the timing for $\tau_{\mathrm{ac}}<\tau_{\mathrm{SN}}$, in other words, the timing for the accretiongrowth activation is well traced by $Z_{\mathrm{c}}$ in Eq. (32) and this is not affected by the uncertainty in $f_{Z}$. On the other hand, the timing for the activation becomes faster for larger $f_{Z}$. The metallicity dependence on the final value of $\delta$ is explicit as found in Eq. (31).

Figure 11 shows the effect of the dust yield. As seen in Fig. 3, our recipe for the stardust yield has a factor of 10 or larger uncertainty because of a large uncertainty in the adopted model calculations. In Fig. 11, we show the cases with a factor of 10 larger or smaller yield than the fiducial one. Other parameters are the same as the fiducial set, so that we have the same evolutions of the metallicity and the time-scales of SN destruction and accretion growth as shown by the solid lines in Fig. 10. Before the growth activation, at around $4 \mathrm{Gyr}$, the dust amounts show a large difference; however, they converge nearly to the same amount after the activation. This is because the final value of $\delta$ given in Eq. (31) does not depend on the dust yield. Therefore, we conclude that the dust content in galaxies is independent of the stardust yield after the grain growth in the ISM becomes active, or equivalently, the metallicity exceeds the critical metallicity. 


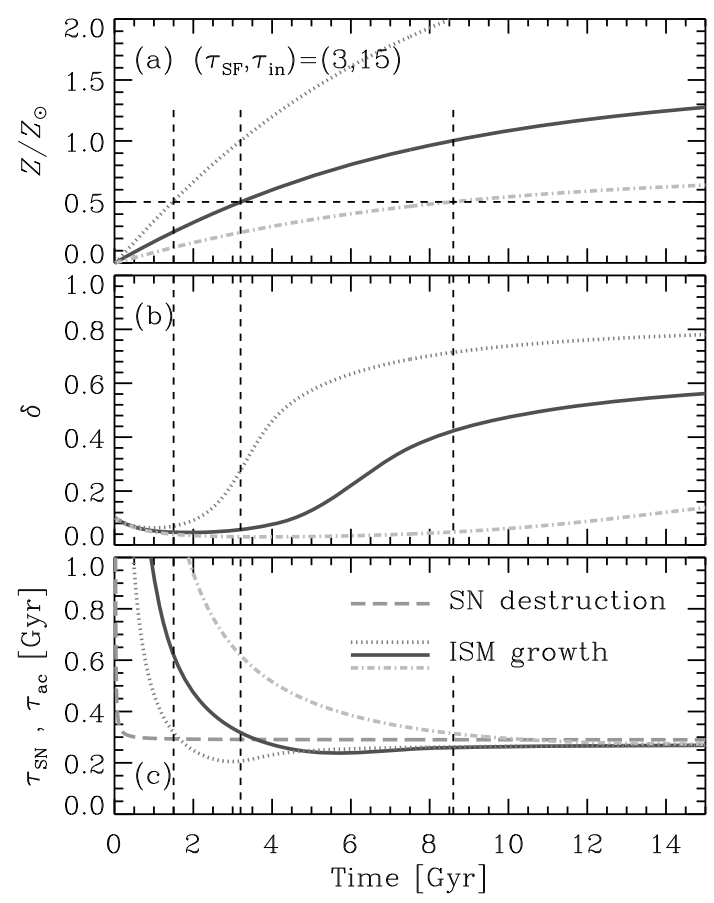

Fig. 10. Time evolution of (a) metallicity, (b) dust-to-metal mass ratio, and (c) time-scales of SN destruction and ISM growth for three different stellar metal yields: $f_{Z}=0.01$ (dot-dashed), 0.02 (solid), and 0.04 (dotted). The horizontal short-dashed line in the panel (a) shows the critical metallicity of Eq. (32). The vertical short-dashed lines indicate the timing at which the metallicity exceeds the critical one for the three metal yields. The long-dashed line in the panel (c) is the SN destruction time-scale, but the other three lines are the ISM growth time-scales for the three metal yields.

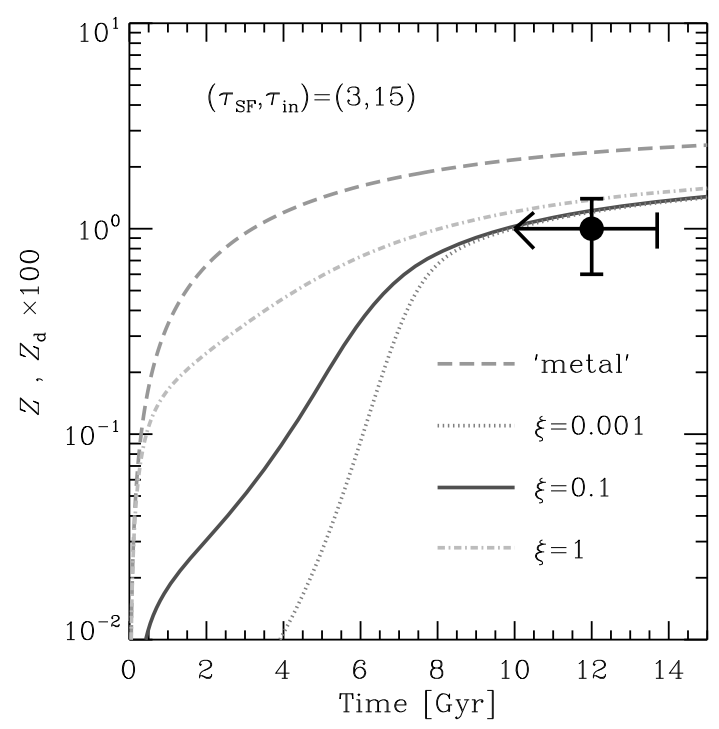

Fig. 11. Same as Fig. 7 but for different condensation efficiencies in the stellar ejecta as indicated in the panel.

\subsection{What kind of dust is formed by the ISM growth?}

We have shown that the main production channel of dust is the accretion growth in the ISM of the present-day Milky Way. This conclusion has also been reported in the literature. For example, Zhukovska et al. (2008) argued that the mass fraction of stardusts in the total dust is only $0.1-1 \%$ based on a more sophisticated chemical evolution model than in this paper (see their figure 15); more than $99 \%$ of dust is originated from accretion growth in the ISM. It is also well known that some interplanetary dust particles show a highly-enhanced abundance of deuterium and ${ }^{15} \mathrm{~N}$ relative to the solar composition, which is a signature of a molecular cloud origin because such isotopic fractionations are expected in a low temperature environment (e.g., Messenger, 2000). Therefore, dust produced by the ISM accretion exists. Then, we have a very important question; what kinds of dust species are formed by the accretion growth in the ISM?

In molecular clouds, many kinds of ices such as $\mathrm{H}_{2} \mathrm{O}$, $\mathrm{CO}, \mathrm{CO}_{2}, \mathrm{CH}_{3} \mathrm{OH}$ have been detected (e.g., Gibb et al., 2000). These ices are condensed onto pre-existent grains. In these ices, some chemical reactions and ultraviolet photolysis (and cosmic rays) process the material and may make it refractory. As a result, so-called 'core-mantle grains' coated by refractory organics would be formed (e.g., $\mathrm{Li}$ and Greenberg, 1997). Indeed, such a grain has been found in cometary dust: olivine particles produced by a Type II SN coated by organic matter which seems to be formed in a cold molecular cloud (Messenger et al., 2005). Therefore, the ISM dust probably has core-mantle or layered structures. Moreover, the composition can be heterogeneous: for example, graphite coated by silicate, silicate coated by graphite, silicate coated by iron, etc. The formation of such grains does not seem to be studied well. Much more experimental and theoretical work is highly encouraged.

If we can find signatures of the dust accretion growth in the ISM of galaxies by astronomical observations (i.e. very distant remote-sensing), it proves the growth to be ubiquitous. Possible evidence already obtained is a huge mass of dust in galaxies which requires accretion growth as discussed in this paper. It is worth studying how to distinguish stardust grains (or grain cores) and ISM dust (or mantle) by observations, e.g., spectropolarimetry, in the future.

\subsection{Dust amount in the proto-solar nebula}

We have shown that the dust amount is very small before ISM growth becomes active. For example, the dust-to-gas mass ratio is of the order of $10^{-4}$ during the first few Gyr following the formation of the Milky Way (or the onset of the major star formation in the solar neighborhood). If the dust-to-gas ratio in the proto-solar nebula was $10^{-4}$, planet formation might have been difficult. Fortunately, the activation of ISM growth is considered to have been about $8 \mathrm{Gyr}$ ago in the solar neighborhood. Thus well before the solarsystem formation. Indeed, we expect a dust-to-gas ratio of several times $10^{-3}$ at 4-5 Gyr ago (see Fig. 7). Moreover, the dust-to-gas ratio may be much enhanced in the protosolar nebula relative to the average ISM. This is because the accretion growth is more efficient at a higher density and the density in the proto-solar nebula is several orders of magnitude higher than that in molecular clouds. Therefore, even if the solar-system formation occurred before the activation of ISM growth globally, dust growth might have been active locally in the proto-solar nebula. In this case, the planet formation is always possible if there is enough metal to accrete onto the pre-existent seed grains, even be- 
fore global growth activation. This is an interesting issue to relate to the Galactic Habitable Zone where complex life can be formed (Lineweaver et al., 2004). We will investigate this more in the future.

Acknowledgments. The author thanks anonymous referees for their many suggestions which were useful to improve the presentation and the quality of this paper. The author is grateful to T. Kozasa and A. Habe for interesting discussions and for their hospitality during my stay in Hokkaido University, Sapporo, where this work was initiated, to R. Asano, H. Hirashita, and T. T. Takeuchi for many discussions, to T. Nozawa for providing his dust yields in $\mathrm{SNe}$, and to $\mathrm{H}$. Kimura, the chair of the convener of the 'Cosmic Dust' session in the AOGS 2010 meeting, for inviting me to the interesting meeting in Hyderabad, India. This work is supported by KAKENHI (the Grant-in-Aid for Young Scientists B: 19740108) by The Ministry of Education, Culture, Sports, Science and Technology (MEXT) of Japan.

\section{References}

Anders, E. and N. Grevesse, Abundances of the elements-Meteoritic and solar, Geochim. Cosmochim. Acta., 53, 197-214, 1989.

Arendt, R. G., E. Dwek, and R. Petre, An infrared analysis of Puppis A, Astrophys. J., 368, 474-485, 1991.

Arendt, R. G., E. Dwek, W. P. Blair, P. Ghavamian, U. Hwang, K. S. Long, R. Petre, J. Rho, and P. F. Winkler, Spitzer observations of dust destruction in the Puppis A supernova remnant, Astrophys. J., 725, 585$597,2010$.

Asano, R., T. T. Takeuchi, H. Hirashita, and A. K. Inoue, Dust formation history of galaxies: a critical role of metallicity for the dust mass growth by accreting materials in the interstellar medium, Earth Planets Space, 2011 (submitted).

Asplund, M., N. Grevesse, A. J. Sauval, and P. Scott, The chemical composition of the sun, Ann. Rev. Astron. Astrophys., 47, 481-522, 2009.

Barlow, M. J., O. Krause, B. M. Swinyard, B. Sibthorpe, M.-A. Besel, R. Wesson, R. J. Ivison, L. Dunne et al., A Herschel PACS and SPIRE study of the dust content of the Cassiopeia A supernova remnant, Astron. Astrophys., 518, L138, 2010.

Bertelli, G., A. Bressan, C. Chiosi, F. Fagotto, and E. Nasi, Theoretical isochrones from models with new radiative opacities, Astron. Astrophys. Suppl., 106, 275-302, 1994.

Bianchi, S. and A. Ferrara, Intergalactic medium metal enrichment through dust sputtering, Mon. Not. R. Astron. Soc., 358, 379-396, 2005.

Bianchi, S. and R. Schneider, Dust formation and survival in supernova ejecta, Mon. Not. R. Astron. Soc., 378, 973-982, 2007.

Borkowski, K. J., B. J. Williams, S. P. Reynolds, W. P. Blair, P. Ghavamian, R. Sankrit, S. P. Hendrick, K. S. Long et al., Dust destruction in Type Ia supernova remnants in the large magellanic cloud, Astrophys. J., 642, L141-L144, 2006.

Calura, F., A. Pipino, and F. Matteucci, The cycle of interstellar dust in galaxies of different morphological types, Astron. Astrophys., 479, 669$685,2008$.

Chabrier, G., Galactic stellar and substellar initial mass function, Publ. Astron. Soc. Pac., 115, 763-795, 2003.

Dopita, M. A. and S. D. Ryder, On the law of star formation in disk galaxies, Astrophys. J., 430, 163-178, 1996.

Draine, B. T., Evolution of interstellar dust, in The Evolution of the Interstellar Medium, 13 pp, Astronomical Society of the Pacific, San Francisco, 1990.

Draine, B. T., Interstellar dust models and evolutionary implications, in Cosmic Dust-Near and Far, edited by Henning, T., E. Grün, J. Steinacker, 20 pp, Astronomical Society of the Pacific, San Francisco, 2009.

Draine, B. T. and E. E. Salpeter, Time-dependent nucleation theory, $J$. Chem. Phys., 67, 2230-2235, 1977.

Draine, B. T. and E. E. Salpeter, Destruction mechanisms for interstellar dust, Astrophys. J., 231, 438-455, 1979.

Dunne, L., S. Eales, R. Ivison, H. Morgan, and M. Edmunds, Type II supernovae as a significant source of interstellar dust, Nature, 424, 285$287,2003$.

Dwek, E., The evolution of the elemental abundances in the gas and dust phases of the galaxy, Astrophys. J., 501, 643-665, 1998.

Dwek, E. and R. G. Arendt, Dust-gas interactions and the infrared emission from hot astrophysical plasmas, Ann. Rev. Astron. Astrophys., 30, 11-50, 1992.

Dwek, E. and I. Cherchneff, The origin of dust in the early universe: Probing the star formation history of galaxies by their dust content, Astrophys. J., 727, 63, 2011.

Dwek, E. and J. M. Scalo, The evolution of refractory interstellar grains in the solar neighborhood, Astrophys. J., 239, 193-211, 1980.

Dwek, E., F. Galliano, and A. P. Jones, The evolution of dust in the early universe with applications to the galaxy SDSS J1148+5251, Astrophys. J., 662, 927-939, 2007.

Dwek, E., R. G. Arendt, P. Bouchet, D. N. Burrows, P. Challis, I. J. Danziger, J. M. De Buizer, R. D. Gehrz et al., Infrared and X-ray evidence for circumstellar grain destruction by the blast wave of Supernova 1987A, Astrophys. J., 676, 1029-1039, 2008.

Edmunds, M. G., An elementary model for the dust cycle in galaxies, Mon. Not. R. Astron. Soc., 328, 223-236, 2003.

Edmunds, M. G. and S. A. Eales, Maximum dust masses in galaxies, Mon. Not. R. Astron. Soc., 299, L29-L31, 1998.

Elmegreen, B. G., Star formation on galactic scales: Empirical laws, in Ecole Evry Schatzman 2010: Star Formation in the Local Universe, Lecture 1 of 5, arXiv:1101.3108, 2011.

Ferrarotti, A. S. and H.-P. Gail, Composition and quantities of dust produced by AGB-stars and returned to the interstellar medium, Astron. Astrophys., 447, 553-576, 2006.

Gall, C., A. C. Andersen, and J. Hjorth, Genesis and evolution of dust in galaxies in the early Universe I. Modeling dust evolution in starburst galaxies, Astron. Astrophys., 528, 13, 2011 a.

Gall, C., A. C. Andersen, and J. Hjorth, Genesis and evolution of dust in galaxies in the early Universe II. Rapid dust evolution in quasars at $z>6$, Astron. Astrophys., 528, 14, 2011 b.

Gehrz, R., Sources of stardust in the galaxy, in Interstellar Dust, edited by Allamandola, L. J. and A. G. G. M. Tielens, 445 pp, International Astronomical Union, Symposium no. 135, Kluwer Academic Publishers, Dordrecht, 1989.

Gibb, E. L. et al., An inventory of interstellar Ices toward the embedded protostar W33A, Astrophys. J., 536, 347-356, 2000.

Gomez, H. L., L. Dunne, R. J. Ivison, E. M. Reynoso, M. A. Thompson, B. Sibthorpe, S. A. Eales, T. M. Delaney, S. Maddox, and K. Isaak, Accounting for the foreground contribution to the dust emission towards Kepler's supernova remnant, Mon. Not. R. Astron. Soc., 397, 16211632, 2009.

Heger, A., C. L. Fryer, S. E. Woosley, N. Langer, and D. H. Hartmann, How massive single stars end their life, Astrophys. J., 591, 288-300, 2003.

Hirashita, H., Global law for the dust-to-gas ratio of spiral galaxies, Astrophys. J., 510, L99-L102, 1999a.

Hirashita, H., Dust-to-gas ratio and phase transition of interstellar medium, Astron. Astrophys., 344, L87-L89, 1999b.

Hirashita, H., Dust-to-gas ratio and metallicity in dwarf galaxies, Astrophys. J., 522, 220-224, 1999c.

Hirashita, H., Dust growth timescale and mass function of molecular clouds in the galaxy, Publ. Astron. Soc. Jpn., 52, 585-588, 2000.

Hirashita, H., Effects of grain size distribution on the interstellar dust mass growth, Mon. Not. R. Astron. Soc., 416, 1340, 2011.

Hirashita, H., Y. Y. Tajiri, and H. Kamaya, Dust-to-gas ratio and star formation history of blue compact dwarf galaxies, Astron. Astrophys., 388, 439-445, 2002.

Inoue, A. K., Evolution of dust-to-metal ratio in galaxies, Publ. Astron. Soc. Jpn., 55, 901-909, 2003.

Inoue, A. K. and H. Kamaya, Constraint on intergalactic dust from thermal history of intergalactic medium, Mon. Not. R. Astron. Soc., 341, L7L11, 2003.

Inoue, A. K. and H. Kamaya, Amount of intergalactic dust: constraints from distant supernovae and the thermal history of the intergalactic medium, Mon. Not. R. Astron. Soc., 350, 729-744, 2004.

Inoue, A. K. and H. Kamaya, Intergalactic dust and its photoelectric heating, Earth Planets Space, 62, 69-79, 2010.

Iwamoto, K., F. Brachwitz, K. Nomoto, N. Kishimoto, H. Umeda, W. R. Hix, and F.-K. Thielemann, Nucleosynthesis in Chandrasekhar mass models for Type IA supernovae and constraints on progenitor systems and burning-front propagation, Astrophys. J. Suppl., 125, 439-462, 1999.

Jenkins, E. B., A unified representation of gas-phase element depletions in the interstellar medium, Astrophys. J., 700, 1299-1348, 2009.

Jones, A. P., A. G. G. M. Tielens, D. J. Hollenbach, and C. F. McKee, Grain destruction in shocks in the interstellar medium, Astrophys. J., 
433, 797-810, 1994.

Jones, A. P., A. G. G. M. Tielens, and D. J. Hollenbach, Grain shattering in shocks: The interstellar grain size distribution, Astrophys. J., 469, 740764, 1996.

Karakas, A. I., Updated stellar yields from asymptotic giant branch models, Mon. Not. R. Astron. Soc., 403, 1413-1425, 2010.

Kennicutt, R. C., The global schmidt law in star-forming galaxies, Astrophys. J., 498, 541-552, 1998.

Kimura, H., I. Mann, and E. K. Jessberger, Composition, structure, and size distribution of dust in the local interstellar cloud, Astrophys. J., 583, 314-321, 2003.

Kozasa, T. and H. Hasegawa, Grain formation through nucleation process in astrophysical environments. II-Nucleation and grain growth accompanied by chemical reaction-, Prog. Theor. Phys., 77, 1402-1410, 1987.

Kozasa, T., T. Nozawa, N. Tominaga, H. Umeda, K. Maeda, and K. Nomoto, Dust in supernovae: Formation and evolution, in Cosmic Dust-Near and Far, edited by Henning, T., E. Grün, and J. Steinacker, 43 pp, Astromomical Society of the Pacific, San Francisco, 2009.

Krause, O., S. M. Birkmann, G. H. Rieke, D. Lemke, U. Klaas, D. C. Hines, and K. D. Gordon, No cold dust within the supernova remnant Cassiopeia A, Nature, 432, 596-598, 2004.

Kroupa, P., The initial mass function of stars: Evidence for uniformity in variable systems, Science, 295, 82-91, 2002.

Larson, R. B., Early star formation and the evolution of the stellar initial mass function in galaxies, Mon. Not. R. Astron. Soc., 301, 569-581, 1998.

Larson, R. B., B. M. Tinsley, and C. N. Caldwell, The evolution of disk galaxies and the origin of S0 galaxies, Astrophys. J., 237, 692-707, 1980.

Li, A. and J. M. Greenberg, A unified model of interstellar dust, Astron. Astrophys., 323, 566-584, 1997.

Lineweaver, C. H., Y. Fenner, and B. K. Gibson, The galactic habitable zone and the age distribution of complex life in the Milky Way, Science, 303, 59-62, 2004.

Lisenfeld, U. and A. Ferrara, Dust-to-gas ratio and metal abundance in dwarf galaxies, Astrophys. J., 496, 145-154, 1998.

Maiolino, R., R. Schneider, E. Oliva, S. Bianchi, A. Ferrara, F. Mannucci, M. Pedani, and M. Roca Sogorb, A supernova origin for dust in a highredshift quasar, Nature, 431, 533-535, 2004.

Matsuura, M., M. J. Barlow, A. A. Zijlstra et al., The global gas and dust budget of the Large Magellanic Cloud: AGB stars and supernovae, and the impact on the ISM evolution, Mon. Not. R. Astron. Soc., 396, 918934, 2009.

Mattsson, L., Dust in the early Universe: Evidence for non-stellar dust production or observational errors?, Mon. Not. R. Astron. Soc., arXiv:1102.0570, 2011 (in press).

McKee, C., Dust destruction in the interstellar medium, in Interstellar Dust, edited by Allamandola, L. and A. G. G. M. Tielens, 14 pp, Kluwer Academic Publishers, Dordrecht, 1989.

Ménard, B., R. Scranton, M. Fukugita, and G. Richards, Measuring the galaxy-mass and galaxy-dust correlations through magnification and reddening, Mon. Not. R. Astron. Soc., 405, 1025-1039, 2010.

Messenger, S., Identification of molecular-cloud material in interplanetary dust particles, Nature, 404, 968-971, 2000.

Messenger, S., L. P. Keller, and D. S. Lauretta, Supernova olivine from cometary dust, Science, 309, 737-741, 2005.

Michałowski, M. J., E. J. Murphy, J. Hjorth, D. Watson, C. Gall, and J. S. Dunlop, Dust grain growth in the interstellar medium of $5<z<6.5$ quasars, Astron. Astrophys., 522, 15, 2010.

Morgan, H. L. and M. G. Edmunds, Dust formation in early galaxies, Mon. Not. R. Astron. Soc., 343, 427-442, 2003.

Morgan, H. L., L. Dunne, S. A. Eales, R. J. Ivison, and M. G. Edmunds, Cold dust in Kepler's supernova remnant, Astrophys. J., 597, L33-L36, 2003.

Mouri, H. and Y. Taniguchi, Grain survival in supernova remnants and Herbig-Haro objects, Astrophys. J., 534, L63-L66, 2000.

Naab, T. and J. P. Ostriker, A simple model for the evolution of disc galaxies: the Milky Way, Mon. Not. R. Astron. Soc., 366, 899-917, 2006.

Nath, B. B., T. Laskar, and J. M. Shull, Dust sputtering by reverse shocks in supernova remnants, Astrophys. J., 682, 1055-1064, 2008.

Nomoto, K., N. Tominaga, H. Umeda, C. Kobayashi, and K. Maeda, Nucleosynthesis yields of core-collapse supernovae and hypernovae, and galactic chemical evolution, Nucl. Phys. A, 777, 424-458, 2006.

Nozawa, T., T. Kozasa, H. Umeda, K. Maeda, and K. Nomoto, Dust in the early universe: Dust formation in the ejecta of population III Super- novae, Astrophys. J., 598, 785-803, 2003.

Nozawa, T., T. Kozasa, and A. Habe, Dust destruction in the high-velocity shocks driven by supernovae in the early universe, Astrophys. J., 648 , 435-451, 2006.

Nozawa, T., T. Kozasa, A. Habe, E. Dwek, H. Umeda, N. Tominaga, K. Maeda, and K. Nomoto, Evolution of dust in primordial supernova remnants: Can dust grains formed in the ejecta survive and Be injected into the early interstellar medium?, Astrophys. J., 666, 955-966, 2007.

Nozawa, T. et al., Formation and evolution of dust in Type IIb supernovae with application to the Cassiopeia A supernova remnant, Astrophys. J., 713, 356-373, 2010.

Onaka, T. and F. Kamijo, Destruction of interstellar grains by sputtering, Astron. Astrophys., 64, 53-60, 1978.

Pagel, B. E. J., The G-dwarf problem and radio-active Cosmochronology, in Evolutionary Phenomena in Galaxies, 23 pp., Cambridge University Press, Cambridge and New York, 1989.

Peacock, J. A., Cosmological Physics, pp. 704, Cambridge University Press, Cambridge, 1999.

Pipino, A., X. L. Fan, F. Matteucci, F. Calura, L. Silva, G. Granato, and R. Maiolino, The chemical evolution of elliptical galaxies with stellar and QSO dust production, Astron. Astrophys., 525, A61, 2011.

Raiteri, C. M., M. Villata, and J. F. Navarro, Simulations of Galactic chemical evolution. I. O and $\mathrm{Fe}$ abundances in a simple collapse model, Astron. Astrophys., 315, 105, 1996.

Rho, J. et al., Freshly formed dust in the Cassiopeia A supernova remnant as revealed by the Spitzer Space Telescope, Astrophys. J., 673, 271-282, 2008.

Rocha-Pinto, H. J., J. Scalo, W. J. Maciel, and C. Flynn, Chemical enrichment and star formation in the Milky Way disk. II. Star formation history, Astron. Astrophys., 358, 869-885, 2000a.

Rocha-Pinto, H. J., W. J. Maciel, J. Scalo, and C. Flynn, Chemical enrichment and star formation in the Milky Way disk. I. Sample description and chromospheric age-metallicity relation, Astron. Astrophys., $\mathbf{3 5 8}$, 850-868, 2000b.

Sakon, I. et al., Properties of newly formed dust by SN 2006JC based on near- to mid-infrared observation with AKARI, Astrophys. J., 692, 546555, 2009.

Salpeter, E. E., The luminosity function and stellar evolution, Astrophys. J., 121, 161-167, 1955

Sankrit, R., B. J. Williams, K. J. Borkowski, T. J. Gaetz, J. C. Raymond, W. P. Blair, P. Ghavamian, K. S. Long, and S. P. Reynolds, Dust destruction in a non-radiative shock in the Cygnus Loop supernova remnant, Astrophys. J., 712, 1092-1099, 2010.

Savage, B. D. and K. R. Sembach, Interstellar abundances from absorption-line observations with the Hubble Space Telescope, Ann. Rev. Astron. Astrophys., 34, 279-330, 1996.

Schmidt, M., The rate of star formation, Astrophys. J., 129, 243-258, 1959.

Schneider, R., A. Ferrara, R. Salvaterra, K. Omukai, and V. Bromm, Lowmass relics of early star formation, Nature, 422, 869-871, 2003.

Schneider, R., A. Ferrara, and R. Salvaterra, Dust formation in very massive primordial supernovae, Mon. Not. R. Astron. Soc., 351, 1379-1386, 2004.

Schneider, R., K. Omukai, A. K. Inoue, and A. Ferrara, Fragmentation of star-forming clouds enriched with the first dust, Mon. Not. R. Astron. Soc., 369, 1437-1444, 2006.

Sibthorpe, B., P. A. R. Ade, J. J. Bock, E. L. Chapin, M. J. Devlin, S. Dicker, M. Griffin, and J. O. Gundersen et al., AKARI and BLAST observations of the Cassiopeia A supernova remnant and surrounding interstellar medium, Astrophys. J., 719, 1553-1564, 2010.

Silvia, D. W., B. D. Smith, and J. M. Shull, Numerical simulations of supernova dust destruction. I. Cloud-crushing and post-processed grain sputtering, Astrophys. J., 715, 1575-1590, 2010.

Songaila, A. and L. L. Cowie, Metal enrichment and ionization balance in the Lyman alpha forest at $z=3$, Astron. J., 112, 335-351, 1996.

Takeuchi, T. T. and H. Hirashita, Testing intermittence of the galactic star formation history along with the infall model, Astrophys. J., 540, $217-$ 223, 2000.

Tielens, A. G. G. M., Interstellar depletions and the life cycle of interstellar dust, Astrophys. J., 499, 267-272, 1998.

Tinsley, B. M., Evolution of the stars and gas in galaxies, Fundam. Cosmic Phys., 5, 287-388, 1980.

Todini, P. and A. Ferrara, Dust formation in primordial Type II supernovae, Mon. Not. R. Astron. Soc., 325, 726-736, 2001.

Valiante, R., R. Schneider, S. Bianchi, and A. C. Andersen, Stellar sources of dust in the high-redshift Universe, Mon. Not. R. Astron. Soc., 397, 1661-1671, 2009. 
van den Bergh, S., The Galaxies of the Local Group, pp. 328, Cambridge University Press, 2000.

Williams, B. J., K. J. Borkowski, S. P. Reynolds, W. P. Blair, P. Ghavamian, S. P. Hendrick, K. S. Long, S. Points et al., Dust destruction in fast shocks of core-collapse supernova remnants in the large magellanic cloud, Astrophys. J., 652, L33-L36, 2006.

Yamamoto, T. and H. Hasegawa, Grain formation through nucleation process in astrophysical environment, Prog. Theor. Phys., 58, 816-828,
1977.

Zhukovska, S., H.-P. Gail, and M. Trieloff, Evolution of interstellar dust and stardust in the solar neighbourhood, Astron. Astrophys., 479, 453480, 2008.

A. K. Inoue (e-mail: akinoue@las.osaka-sandai.ac.jp) 